\title{
Emergency Department MRI Scanning of Patients with Multiple Sclerosis: Worthwhile or Wasteful?
}

\author{
(D). Pakpoor, (DD. Saylor, (DI. Izbudak, (D) L. Liu, (D) E.M. Mowry, and (DD.M. Yousem
}

\begin{abstract}
BACKGROUND AND PURPOSE: The increasing use of the emergency department MR imaging scanner at our institution raises questions about its added value to certain patient groups. We hypothesized that the use of emergency department MR imaging for identifying active demyelination in MS patients presenting with new neurologic symptoms would be of low yield.
\end{abstract}

MATERIALS AND METHODS: Electronic medical records were reviewed for patients with MS who had emergency department MR imaging scans for a suspected MS exacerbation between March 1, 2014, and March 1, 2016. Details surrounding patient disposition, imaging, diagnosis, and management were determined.

RESULTS: Of 115 patients in our study, 48 (41.7\%) were ultimately diagnosed with an MS exacerbation. Nearly all patients with MS exacerbations $(87.5 \%, 42 / 48)$ had active demyelination on their emergency department MR imaging, identified on 30.6\% (33/108) of brain MRIs and $20.4 \%$ (19/93) of spinal MRIs. The presence of active demyelination at MRI was significantly associated with the ultimate diagnosis of an MS exacerbation $(P<.001)$. MR imaging activity isolated to the spinal cord (ie, not found on concurrent brain MR imaging) was present in only 9 of $93(9.7 \%)$ cases. Pseudoexacerbations accounted for 18 of the alternative diagnoses.

CONCLUSIONS: Emergency department MR imaging is a worthwhile endeavor from a diagnostic standpoint for MS exacerbations despite not being part of the diagnostic criteria. This finding has corresponding downstream impact on management decisions to admit and/or administer intravenous steroids. However, we raise the question of whether clinicians over-rely on emergency department imaging for making exacerbation diagnoses. Additionally, spinal MR imaging is of questionable value as an addition to brain MR imaging due to a low yield of isolated spinal disease.

ABBREVIATIONS: $\mathrm{AD}=$ active demyelination; $\mathrm{ED}=$ emergency department

M $\mathrm{S}$ is a chronic demyelinating disorder of the CNS affecting an estimated 2.5 million individuals worldwide. ${ }^{1}$ Approximately $80 \%$ of individuals with MS initially present with the relapsing-remitting form of the disease, characterized by intermittent episodes of exacerbations of a variety of neurologic symptoms that can reflect underlying acute inflammation (eg, numbness or tingling of the limbs, blurred vision, and weakness). ${ }^{2-6}$ Exacerbations are generally not life-threatening, and neurologic recovery can be hastened with high-dose steroids. ${ }^{7-10}$ However, the unpredictable timing of their occurrence and vari-

Received June 10, 2016; accepted after revision August 11.

From the Division of Neuroradiology (J.P., I.I., L.L., D.M.Y.), Russell H. Morgan Department of Radiology and Radiological Science, and Department of Neurology (D.S., E.M.M.), Johns Hopkins Medical Institutions, Baltimore, Maryland.

Please address correspondence to David M. Yousem, MD, MBA, Division of Neuroradiology, Russell H. Morgan Department of Radiology and Radiological Science, Johns Hopkins Medical Institutions, 600 N Wolfe St, Phipps B100F, Baltimore, MD 21287; e-mail: dyousem1@jhu.edu

http://dx.doi.org/10.3174/ajnr.A4953 ability of neurologic deficits may lead patients to present to the emergency department (ED). A 2014 study at the Mount Sinai Hospital ED in New York found an average of 2.5 visits to the ED per patient with MS for neurologic and non-neurologic problems during a 3-year period. ${ }^{11}$

Currently, MR imaging is the preferred tool for assessing MS disease activity. "MR imaging activity," defined as gadoliniumenhancing lesions and/or lesions with restricted diffusion, is used as a marker of active demyelination (AD) during an exacerbation. ${ }^{12-17}$ New or enlarging T2WI/FLAIR hyperintense lesions can also be used as indicators of disease progression but are generally considered less sensitive for the assessment of acute activity. ${ }^{15}$ While MR imaging can therefore be useful for confirming MS exacerbations, it is costly; of long duration when scanning the brain and cervicothoracic spine; technically challenging so as not to miss lesions, particularly in the thoracic spine; and not part of the diagnostic criteria for MS exacerbations. ${ }^{7,12-14,18,19}$ The diagnostic criteria have been defined by the International Panel on the 
Diagnosis of MS to be neurologic signs typical of an acute inflammatory demyelinating event in the CNS, of at least 24-hour duration. $^{8}$

In April 2012, an MR imaging scanner was installed in the ED of our hospital for use 24 hours per day. The following year saw a $51.4 \%$ increase in MR imaging use in the ED for neurology consult patients. ${ }^{20}$ While this reduced the burden on the inpatient MR imaging scanners, the increased use of ED MR imaging raised the question of its added value to certain patient groups for what is still a limited but heavily used hospital resource. Additionally, the use of emergency imaging has previously been shown to be associated with increased patient length of stay in the ED and downstream ED crowding, reduced patient satisfaction, and negative impacts on the timeliness of patient care. ${ }^{20-24}$ A 2012 study that used ED data from a national data base found MR imaging use to be associated with a 64-minute increase in the length of stay in the ED, second only to blood tests for time-cost among testing modalities. $^{23}$

This study aimed to assess the value of using the ED MR imaging scanner for patients with a known diagnosis of MS by evaluating the extent to which it is used as a diagnostic tool for MS exacerbations and its yield of diagnostic findings. On the basis of the anecdotal experience of the radiologist authors, we hypothesized the following: 1) that MS exacerbations identified by $\mathrm{AD}$ plaques would occur in less than half (50\%) of cases, 2$)$ suggesting an alternative diagnosis to MS exacerbation based on an MR imaging finding would be an uncommon $(<10 \%)$ occurrence, 3$)$ the management decision of the treating physicians would rarely be affected ( $<5 \%$ of ED presentations), and 4$)$ new enhancing spinal cord lesions would rarely be detected (ie, $<5 \%$ of patients).

\section{MATERIALS AND METHODS}

This study complied with the Health Insurance Portability and Accountability Act and was approved by the institutional review board at our institution. Because of its retrospective nature, informed consent requirements were waived.

\section{Data Collection and Analysis}

An automated query of the radiology information system of our institution was used to identify all patients with ED MR imaging encounters in a 2-year period, March 1, 2014, to March 1, 2016, in which at least 1 of the terms "multiple sclerosis," "MS," "clinically isolated syndrome," "demyelination," "demyelinating," "myelitis," or "optic neuritis" appeared anywhere in the ED MR imaging report. The total proportion of $\mathrm{ED}$ neuroradiologic $\mathrm{MRl}$ requests that were for suspected MS exacerbations was determined by searching the indications of all Current Procedural Terminology codes for neuroradiology procedures in the ED MR scanner during that period. We then limited our study to those patients with a preexisting diagnosis of MS who had presented to the ED and for whom an "MS exacerbation" was part of the differential diagnosis in the patients' electronic medical records. An MS exacerbation could be indicated by synonymous terminology (eg, "flare-up," "attack," or "relapse"). For each encounter, patient demographics, presenting symptoms, imaging protocol used, MR imaging radiology report, final diagnosis, disposition (admission versus discharge), treatment (high-dose steroids or no steroids), and
Table 1: ED MRI requesting patterns by CNS location imaged

\begin{tabular}{lcc}
\hline \multicolumn{1}{c}{ Encounter MRI Requests } & N = 115 & \% of Total \\
\hline Brain & 17 & $14.5 \%$ \\
Brain + orbit & 4 & $3.5 \%$ \\
Brain + C-spine & 9 & $7.8 \%$ \\
Brain + orbit + C-spine & 1 & $0.9 \%$ \\
Brain + C- + T-spine & 53 & $46.1 \%$ \\
Brain + orbit + C- + T-spine & 2 & $1.8 \%$ \\
Brain + C- + T- + L-spine & 21 & $18.3 \%$ \\
Brain + orbit + C- + T- + L-spine & 1 & $0.9 \%$ \\
Orbit & 1 & $0.9 \%$ \\
C-spine & 1 & $0.9 \%$ \\
T- + C-spine & 4 & $3.5 \%$ \\
T- + C- + L-spine & 1 & $0.9 \%$ \\
\hline
\end{tabular}

Note:- $C$ indicates cervical; $T$, thoracic; L, lumbar.

length of stay (if admitted) were determined. Additionally, we reviewed the electronic medical records to determine the proportion of patients for whom the neurology consult recommended MR imaging.

For comparison, ED admission records were also queried for patients with MS admitted to the neurology department for a suspected MS exacerbation from the ED without obtaining an ED MR image. For these patients, we examined whether they subsequently underwent inpatient MR imaging and their length of stay on admission. Per hospital procedure, it is highly unlikely that a patient with MS presenting to the ED with a suspected MS exacerbation would be discharged from the ED without either admission for management or inpatient or ED imaging; thus such cases were not included in this study.

\section{ED MR Imaging Requests}

Of the 115 patients who were scanned in the ED, 93.0\% (107/115) had neuroradiological MR imaging through use of the hospital's MS protocol with and without gadolinium contrast administration (as opposed to the stroke or routine brain protocols). This protocol includes a 6-minute 3D FLAIR whole-brain pulse sequence in the sagittal plane, which could be reconstructed in axial and coronal sections. Of the $115,69.6 \%(n=80)$ had a combination of MR imaging of the brain and spine under the MS protocol. A further 18 patients had only MR imaging of the brain under the MS protocol, and 9 patients had only MR imaging of the spine under the MS protocol. Eight patients had imaging under the routine brain or stroke protocols.

In total, $108 \mathrm{MR}$ images of the brain, 93 of the cervical spine, 82 of the thoracic spine, 22 of the lumbar spine, and 9 orbit examinations were obtained. The CNS locations imaged by using the ED MR imaging scanner and the combinations used for each encounter are represented in Table 1.

\section{Statistical Analyses}

We calculated a Pearson $\chi^{2}$ test of independence, comparing the frequency of identification of MR imaging activity and the diagnosis of an MS exacerbation. A Wilcoxon rank sum test was used to compare the length of stay on admission of patients who had ED MR imaging versus inpatient MR imaging. Statistical significance was defined at $P<.05$. Analyses were performed by using STATA (StataCorp, College Station, Texas). 


\section{RESULTS}

Between March 1, 2014, and March 1, 2016, 4\% of all neuroradiologic ED MR imaging requests (MR imaging of the brain, orbits, cervical, thoracic, and lumbar spine) were for patients with an established MS diagnosis who presented to the ED with a possible MS exacerbation. These represent the 115 ED encounters of patients with MS who made up our study population. Their demographic profiles are shown in Table 2.

For $74.8 \%$ (86/115) of patient encounters, it was determined from the patients' electronic medical records that the MR imaging requests were recommended by a neurology resident consult, not solely by the ED service itself.

\section{ED MR Imaging and Diagnosis}

Of 115 patients with MS in our study population, $41.7 \%(n=48)$ were diagnosed with an MS exacerbation. Their findings at ED MR imaging are shown in Table 3.

Of the 42 patients who were diagnosed with an MS exacerbation and who had MR imaging activity, 37 had gadoliniumenhancing lesions, 3 had diffusion restriction and gadoliniumenhancing lesions, and 2 had only lesions of diffusion restriction. Two patients with alternative clinical diagnoses also had lesions of diffusion restriction.

\section{MR Imaging Activity and CNS Location}

The breakdown of the CNS locations where MR imaging activity was identified is shown in Table 4 . The 33 patients with demonstrated MR imaging brain activity accounted for $78.6 \%$ of the 42 patients who had such activity demonstrated. Three-quarters of encounters (85/115) included both MR imaging studies of the brain and cervical spine, and in only $7.1 \%$ (6/85) of these cases did MR imaging of the cervical spine demonstrate MR imaging activity when the MR imaging of the brain did not. Among the 82 cases with thoracic spine MR imaging, it was the only location to demonstrate AD in only 3 cases (3.7\%). In total, 20.4\% (19/93) of encounters that used any spinal imaging demonstrated MR imaging activity in the spine, but spine imaging demonstrated MR imaging activity in the absence of activity in the brain in $<10 \%$ (9/93, 9.7\%) of cases.

An MS exacerbation was significantly more likely to be diagnosed if the MR imaging showed activity compared with patients in whom this finding was lacking $(P<.001$, odds ratio $=462$; 95\% confidence interval, 128-1670).

Table 2: Patient demographic profiles

\begin{tabular}{lc}
\hline Demographics & \\
\hline No. & 115 \\
Age (yr) & $39 \pm 12.1$ \\
Sex & 98 Women, 17 men \\
\hline
\end{tabular}

In the 67 patients who were not diagnosed with an MS exacerbation, a positive finding other than $\mathrm{AD}$ on $\mathrm{MR}$ imaging suggested an alternative diagnosis in only 7.5\% (5/67) of cases. These were C5 spondyloradiculopathy, tonsillitis, degenerative compressive myelopathy $(n=2)$, and stroke. Final diagnoses in patients not diagnosed with an MS exacerbation varied considerably (Table 5).

\section{Management}

Admission and Treatment. In total, 59.1\% (68/115) of our study population were recommended for admission, and 91.7\% (44/48) of those with a final diagnosis of an MS exacerbation were admitted. All patients admitted with an MS exacerbation were treated with intravenous methylprednisolone except 1 patient who had a previous adverse reaction to high-dose steroids. Of patients not diagnosed with an MS exacerbation, 35.8\% (24/67) required admission for their alternative diagnoses. These included neurosurgical intervention for decompression, symptomatic pain relief and fluid management, antibiotics for tonsillitis, and so forth. On the basis of review of the electronic medical records, the decision as to whether to admit the patient correlated with identification or absence of $\mathrm{AD}$ lesions in 70\% of all encounters (80/115). Neurology consult notes in the medical records of 39\% (45/115) of the MS patients in the ED also indicated that MR imaging findings would determine disposition (admission versus discharge) and treatment (high-dose steroids or not).

In the same time period, 19 patients with MS were admitted to the neurology department for a suspected MS exacerbation without having ED MR imaging. Except for 1 patient who refused due to pregnancy, the other 18 had MR imaging as inpatients (Table 6). Eight of these patients were ultimately diagnosed with an MS exacerbation, 3 of whom did not demonstrate MR imaging activity. Eleven were admitted for an MS exacerbation but had an alternative final diagnosis established. The difference in length of stay upon admission between patients with MS admitted following ED MR imaging (median, 3.3 days; interquartile range, 1.94.4 days) versus with subsequent inpatient imaging (median, 4.2 days; interquartile range, 2.1-6.4 days) was not statistically significant because of the large SDs $(P>.05)$. Nonetheless, the average hospital admission with ED MR imaging was 0.9 days shorter than if admitted without such imaging. In 2 of 11 of these patients who were not given a final diagnosis of an MS exacerbation, a 5-day course of IV methylprednisolone was commenced before the availability of inpatient MR imaging results, and was subsequently stopped midcourse as an alternative diagnosis was made.

No patient with MS with a suspected MS exacerbation who presented to the ED in the 2-year period of the study was admitted

Table 3: Findings at MRI in patients diagnosed with an MS exacerbation or an alternative diagnosis

\begin{tabular}{|c|c|c|c|c|c|}
\hline & $\begin{array}{l}\text { MRI Activity Only } \\
\text { (with Enhancement or }^{\text {Diffusion Restriction) }}\end{array}$ & $\begin{array}{l}\text { MRI Activity + } \\
\text { Progressive WM } \\
\text { Lesions }\end{array}$ & $\begin{array}{l}\text { Progressive WM Lesions } \\
\text { Only (without Enhancement } \\
\text { or Diffusion Restriction) }{ }^{\mathrm{b}}\end{array}$ & $\begin{array}{l}\text { MRI } \\
\text { Negative for } \\
\text { MS Change }\end{array}$ & Total \\
\hline $\begin{array}{l}\text { Final clinical diagnosis of MS exacerbation } \\
\text { (No.) }\end{array}$ & 15 & 27 & 1 & 5 & 48 \\
\hline Other final clinical diagnoses (No.) & 0 & 1 & 12 & 54 & 67 \\
\hline
\end{tabular}

a The presence of a sign of active demyelination (ie, the presence of gadolinium-enhancing lesions and/or diffusion restriction).

b The reporting of a new or enlarging white matter lesion. 
Table 4: CNS location and MRI activity ${ }^{a}$

\begin{tabular}{lccccc}
\hline & \multicolumn{3}{c}{ Cervical } & Lumbar \\
Imaging Section & Brain & Spine & Thoracic & Spine & Orbit \\
\hline MRI activity & $33(31 \%)$ & $13(14 \%)$ & $8(10 \%)$ & $0(0 \%)$ & $4(44 \%)$ \\
No MRI activity & $75(69 \%)$ & $80(86 \%)$ & $74(90 \%)$ & $22(100 \%)$ & $5(56 \%)$ \\
Total & 108 & 93 & 82 & 22 & 9 \\
\hline
\end{tabular}

a Percentages of column totals are in parentheses.

Table 5: Number of alternative diagnoses made for patients not diagnosed with an MS exacerbation in the study population Diagnosis

No final diagnosis: 23

Pseudoexacerbation: 18

Neuropathic pain: 5

Musculoskeletal: 5

Migraine: 2

Seizure: 2

Neuralgia: 3

Compressive myelopathy: 2

Arthralgia: 1

Tonsillitis: 1

Avascular necrosis: 1

C5 radiculopathy: 1

Postconcussive syndrome: 1

Stroke: 1

Acute manic bipolar disorder episode: 1

Table 6: MRI findings at inpatient MRI

\begin{tabular}{lccccc}
\hline & Brain & Cervical & Thoracic & Lumbar & Orbit \\
\hline Total scans (No.) & 16 & 14 & 14 & 3 & 1 \\
MRI activity (No.) & 5 & 0 & 1 & 0 & 0 \\
Progressive WM & 2 & 0 & 0 & 0 & 0 \\
$\quad$ lesions (No.) & & & & & \\
\hline
\end{tabular}

for IV steroid treatment without an MR imaging study at some point in their hospitalization.

\section{DISCUSSION}

A growing number of hospital EDs may be compelled to acquire MR imaging scanners as they strive to meet the requirements of The Joint Commission Standards for Comprehensive Stroke Center certification, which currently necessitate around-the-clock availability of on-site MR imaging. ${ }^{25}$ It is therefore of increasing relevance to establish the utility of ED MR imaging for various patient groups. In the 2-year period encompassed by our study, patients with MS with suspected MS exacerbations represented $4 \%$ of neuroradiologic MR imaging requests that used the ED scanner. Before the introduction of MR imaging in the ED, these and other patients requiring MR imaging during their ED encounter would require the approval of a radiologist and transport to a more remote area of the hospital (estimated travel time, 5-10 minutes). A technologist would be called in emergently between the hours of 11 PM and 6 AM for off-shift scanning.

We found that around one-third (36.5\%) of MS encounters in our study population were diagnosed with an MS exacerbation with demonstration of MR imaging activity on ED MR imaging, in keeping with our first hypothesis that MR imaging activity in this patient population would be identified $<50 \%$ of cases. The presence of gadolinium-enhancing lesions was significantly associated with a diagnosis of an MS exacerbation because these were present in $92.5 \%$ of cases diagnosed as such, versus only in 1 case of a patient with MS with an alternative diagnosis $(P<.001)$. Of the diagnosed MS exacerbations, more than half (58.3\%) also demonstrated new or enlarging hyperintense lesions at MR imaging to support disease activity. Although we further hypothesized that the likelihood of suggesting an alternative diagnosis to an MS exacerbation based on a positive MR imaging finding would be an uncommon occurrence (ie, less than $10 \%$ of encounters), we found that MR imaging findings provided alternative diagnoses in only $7.5 \%$ of cases $(n=5)$. Most $(60 \%)$ encounters not diagnosed as MS exacerbations either had no final diagnosis established or documented (23 encounters) or were diagnosed as pseudoexacerbations (18 encounters). The latter are instances in which heat, stress, or infection can cause a recurrence of MS symptoms from a previous exacerbation without underlying new inflammation or disease progression (as such these have no pathognomonic findings on MR imaging and are not treated with IV steroids). ${ }^{26,27}$

Our third hypothesis was that the management decision of the treating physician would rarely be affected by MR imaging ( $<10 \%$ of cases). However, in $70 \%$ of encounters, the decision as to whether to admit the patient correlated with identification of $\mathrm{AD}$ lesions. This is explained by the significant relationship between identification of MRI imaging activity and an MS exacerbation diagnosis. Our findings suggest that while ED MR imaging has a low yield in identifying alternative diagnoses in this patient group, ruling out an MS exacerbation can avoid unnecessary hospitalization for intravenous steroid administration and its associated cost. This finding is supported by the commencement and early termination of IV methylprednisolone treatment in 2 patients in our comparison group who were admitted without ED MR imaging and were subsequently not diagnosed with MS exacerbations after inpatient scans. The difference in the length of stay on admission of patients who had ED MR imaging versus inpatient MR imaging was not statistically significant; however, the difference in the median length of stay was 0.9 days, a notable cost. Furthermore, only 8/19 (42.1\%) of those patients admitted without an ED MR imaging for presumed MS exacerbation actually had the diagnosis. This finding contrasts with that in those patients having preceding ED MR imaging: 91.7\% (44/48) of those with a final diagnosis of an MS exacerbation were admitted. These 44 patients represent $64.7 \%$ (44/68) of admissions in the population examined, much better than without the ED MR imaging.

The significant association between MR imaging activity and the diagnosis of an exacerbation (and therefore subsequent management decisions) appears to suggest that ED MR imaging may be worthwhile from a diagnostic standpoint for suspected MS exacerbations. However, the association raises 2 important issues that warrant further consideration.

The first issue is whether studies of different CNS locations have equal diagnostic value. While the proportion of spinal MRIs that demonstrated enhancing lesions was greater than hypothesized (20.4\%), AD shown on MR imaging was limited to the spine in only $9.7 \%$ of cases (ie, MR imaging activity was not concomitantly found on MR imaging of the brain). Nonetheless, approximately two-thirds of patients had a combination of MR imaging of the brain and the cervical and thoracic spine ordered as a set from the ED. In only $7 \%$ of these instances (6/85) was the cervical spine the only imaging location to show MR imaging activity. The 
value of thoracic spine MR imaging was particularly low considering that it was requested for $71 \%$ of patients with MS but was the sole MR imaging examination that showed MR imaging activity in only $4 \%$ of these encounters. The study of all 3 body parts for nearly three-quarters of our patient group must therefore be questioned in light of the low yield of spinal MR imaging as the only location of MR imaging activity. Our results suggest that a more cost-efficient MR imaging request paradigm may be represented by performing an MR imaging of the brain initially and requesting further spinal images only as needed subsequently. The management of an MS exacerbation in the form of steroid treatment is standard irrespective of the site of the exacerbation. The disadvantage of such a paradigm may be time-cost in the patient potentially requiring multiple separate MR imaging studies. However, having a radiologist review the brain MR imaging while the patient is still in the MR imaging scanner to see if additional imaging is required could avoid this limitation. Although the proportion of requested MR imaging of lumbar spine scans was relatively low ( $20 \%$ of encounters), these identified no findings of diagnostic value in our study population.

The second major issue that arises from these results is whether there is an over-reliance on identification of MR imaging activity as a diagnostic determinant of an MS exacerbation, even though MR imaging findings are not part of the diagnostic criteria for an MS exacerbation. If MR imaging findings are the major determining factor in whether a patient is diagnosed with an MS exacerbation, this would bias our analysis of the utility of ED MR imaging. We found that for 39\% of patients (45/115), the electronic medical records explicitly indicated that a management decision to admit and/or administer intravenous steroids for an MS exacerbation was determined by findings of MR imaging activity. For example, documentation from 1 patient's neurology consultation record stated, "Steroids/admission if evidence of enhancement, otherwise discharge with outpatient follow-up." Because MR imaging results were available before neurology consultation for a substantial proportion of the study population, we suspect that our figure of $39 \%$ is actually an underestimation of the role of MR imaging in the decision-making process. These findings indicate reliance on demonstration of MR imaging activity to make a diagnosis of an MS exacerbation when patients present to the ED. Further evidence toward reliance on MR imaging is that no (nonpregnant) patient with MS presenting to the ED was admitted for treatment of an MS exacerbation without either an ED MR imaging or inpatient MR imaging, despite the current definition of MS exacerbations as a clinical diagnosis.

The use of brain and spinal ED MR imaging for making the diagnosis of an exacerbation may reflect a lack of experience or confidence among the neurology trainees and non-neurology specialists who perform the initial evaluations of these patients in the ED. In addition, the availability of an MR imaging scanner in the ED itself could be contributing to an unintentional overuse and over-reliance on such imaging. A lack of specialist experience by the physicians, heavy patient loads, staff shortages, and stringent time pressures may provoke the substitution of imaging for other clinical assessments. ${ }^{28-30}$ An over-reliance can be problematic both due to unnecessary use of an expensive resource but also because current imaging methods for detect- ing $\mathrm{AD}$ are not $100 \%$ sensitive and thus may result in underdiagnosis of MS exacerbations. ${ }^{5}$

Further research is needed in a prospectively designed study to assess whether physicians would reach the same diagnostic decisions if they did not have access to ED MR imaging. Additionally, research should explore differences in MR imaging use by the level of physician experience, including discrepancies between neurologists, neurology trainees, and MS specialists.

Finally, this study should be viewed in the context of a number of limitations. Variability in note-taking by multiple physicians means that objective measures of MR imaging reliance were limited. This was a single-institution study at an academic medical center, so all of the study patients were initially assessed by physicians-in-training. The ability to perform statistical analyses comparing patients who had an ED MR imaging versus inpatient MR imaging was limited by the small sample size of patients in the latter group, resulting in a skewed dataset and power of only $26 \%$ at the $5 \%$ significance level. It would be of interest to further analyze discrepancies in a larger patient set. For instance, our results demonstrate that the percentage of patients with positive findings on MR imaging may be higher in patients with ED-versus-inpatient MR imaging.

This study is also limited by its retrospective study design, which means that we cannot determine what the physicians would or would not have done without the availability of MR imaging, and MR imaging results may be biasing the clinical diagnoses. This study only examined use for patients with a known MS diagnosis. The full use of ED MR imaging for MS diagnoses (ie, including for those patients not yet fulfilling the McDonald criteria for MS) was not examined in this cohort. ${ }^{14}$ Finally, we were not able to determine retrospectively the downstream impact of ED crowding when the MR imaging scanner was used, such as delays for other patients while the relatively long braincervical-thoracic spine MR imaging was taking place. For example, whether this impacts imaging availability for patients presenting with symptoms concerning for an acute stroke is a useful future research area for a prospective study.

\section{CONCLUSIONS}

ED MR imaging is a worthwhile endeavor from a diagnostic standpoint to assess for an MS exacerbation and has corresponding downstream impact on management decisions to admit patients and administer intravenous steroids. However, we raise the possibility that physicians may be placing undue weight on MR imaging findings relative to history and physical examination findings in diagnosing MS exacerbations. An examination of this and a full cost-benefit analysis of ED MR imaging for suspected MS exacerbations represent important avenues for future work. We further found that immediate concurrent spinal MR imaging use is a questionable addition to MR imaging of the brain due to its particularly low yield in identifying isolated spinal $\mathrm{AD}$, especially in the thoracic and lumbar spine.

Disclosures: Izlem Izbudak—UNRELATED: Grants/Grants Pending: Siemens*; Other: Biogen, Comments: pending*. Ellen M. Mowry-UNRELATED: Grants/Grants Pending: Biogen, Sun Pharma, Comments: investigator-initiated trial from Biogen, site Principal Investigator of trials from Biogen and Sun Pharma*; Royalties: UptoDate, Comments: chapter editing; Other: Teva Neuroscience, Comments: free medi- 
cation for clinical trial, of which I am the Principal Investigator*. David M. Yousem-UNRELATED: Medicolegal neuroradiologist expert testimony. Payment for Lectures including service on Speakers Bureaus: American College of Radiology Education Center; Royalties: book royalties for 4 books. Payment for Development of Educational Presentations: CMEinfo.com*. *Money paid to the institution.

\section{REFERENCES}

1. The National Multiple Sclerosis Society. http://www.nmss.org/. Accessed June 10, 2016

2. Lublin FD, Reingold SC. Defining the clinical course of multiple sclerosis: results of an international survey-National Multiple Sclerosis Society (USA) Advisory Committee on Clinical Trials of New Agents in Multiple Sclerosis. Neurology 1996;46:907-11 CrossRef Medline

3. Racke MK. Immunopathogenesis of multiple sclerosis. Ann Indian Acad Neurol 2009;12:215-20 CrossRef Medline

4. Compston A, Coles A. Multiple sclerosis. Lancet 2008;372:1502-17 CrossRef Medline

5. Lublin FD. The incomplete nature of multiple sclerosis relapse resolution. J Neurol Sci 2007;256:S14-18 CrossRef Medline

6. Frohman EM, Eagar T, Monson N, et al. Immunologic mechanisms of multiple sclerosis. Neuroimaging Clin N Am 2008;18:577-88, ix CrossRef Medline

7. Ontaneda D, Rae-Grant AD. Management of acute exacerbations in multiple sclerosis. Ann Indian Acad Neurol 2009;12:264-72 CrossRef Medline

8. Polman CH, Reingold SC, Banwell B, et al. Diagnostic criteria for multiple sclerosis: 2010 revisions to the McDonald criteria. Ann Neurol 2011;69:292-302 CrossRef Medline

9. Repovic P, Lublin FD. Treatment of multiple sclerosis exacerbations. Neurol Clin 2011;29:389-400 CrossRef Medline

10. Burton JM, O'Connor PW, Hohol M, et al. Oral versus intravenous steroids for treatment of relapses in multiple sclerosis. Cochrane Database Syst Rev 2012;12:CD006921 CrossRef Medline

11. Oynhausen S, Alcauskas M, Hannigan C, et al. Emergency medical care of multiple sclerosis patients: primary data from the Mount Sinai resource utilization in multiple sclerosis project. J Clin Neurol 2014;10:216-21 CrossRef Medline

12. Arnold DL, Matthews PM. MRI in the diagnosis and management of multiple sclerosis. Neurology 2002;58:S23-31 CrossRef Medline

13. Karussis D. The diagnosis of multiple sclerosis and the various related demyelinating syndromes: a critical review. J Autoimmun 2014;48-49:134-42 CrossRef Medline

14. McDonald WI, Compston A, Edan G, et al. Recommended diagnostic criteria for multiple sclerosis: guidelines from the International Panel on the Diagnosis of Multiple Sclerosis. Ann Neurol 2001;50: 121-27 CrossRef Medline

15. Bonzano L, Roccatagliata L, Mancardi GL, et al. Gadolinium-enhancing or active T2 magnetic resonance imaging lesions in multi- ple sclerosis clinical trials? Mult Scler 2009;15:1043-47 CrossRef Medline

16. Nusbaum AO, Lu D, Tang CY, et al. Quantitative diffusion measurements in focal multiple sclerosis lesions: correlations with appearance on TI-weighted MR images. AJR Am J Roentgenol 2000;175: 821-25 CrossRef Medline

17. Kornienko VN, Pronin IN. Diagnostic Neuroradiology. Berlin Springer Berlin; 2009

18. Kermode AG, Tofts PS, Thompson AJ, et al. Heterogeneity of bloodbrain barrier changes in multiple sclerosis: an MRI study with gadolinium-DTPA enhancement. Neurology 1990;40:229-35 CrossRef Medline

19. Hyland M, Bermel RA, Cohen JA. Restricted diffusion preceding gadolinium enhancement in large or tumefactive demyelinating lesions. Neurol Clin Pract 2013;3:15-21 CrossRef Medline

20. Redd V, Levin S, Toerper M, et al. Effects of fully accessible magnetic resonance imaging in the emergency department. Acad Emerg Med 2015;22:741-49 CrossRef Medline

21. Bernstein SL, Aronsky D, Duseja R, et al; Society for Academic Emergency Medicine, Emergency Department Crowding Task Force. The effect of emergency department crowding on clinically oriented outcomes. Acad Emerg Med 2009;16:1-10 CrossRef Medline

22. Gardner RL, Sarkar U, Maselli JH, et al. Factors associated with longer ED lengths of stay. Am J Emerg Med 2007;25:643-50 CrossRef Medline

23. Kocher KE, Meurer WJ, Desmond JS, et al. Effect of testing and treatment on emergency department length of stay using a national database. Acad Emerg Med 2012;19:525-34 CrossRef Medline

24. Trzeciak S, Rivers EP. Emergency department overcrowding in the United States: an emerging threat to patient safety and public health. Emerg Med J 2003;20:402-05 CrossRef Medline

25. Advanced Disease-Specific Care Certification Requirements for Comprehensive Stroke Center (CSC). http://www.jointcommission. org/assets/1/18/dsc_csc_chap.pdf. Accessed May 16, 2016

26. Thrower BW. Relapse management in multiple sclerosis. Neurologist 2009;15:1-5 CrossRef Medline

27. Wingerchuk DM, Rodriguez M. Premenstrual multiple sclerosis pseudoexacerbations: role of body temperature and prevention with aspirin. Arch Neurol 2006;63:1005-08 CrossRef Medline

28. American Academy of Pediatrics Committee on Pediatric Emergency Medicine. Overcrowding crisis in our nation's emergency departments: is our safety net unraveling? Pediatrics 2004;114: 878-88 CrossRef Medline

29. Derlet RW, Richards JR. Overcrowding in the nation's emergency departments: complex causes and disturbing effects. Ann Emerg Med 2000;35:63-68 CrossRef Medline

30. Zwemer FL Jr. Emergency department overcrowding. Ann Emerg Med 2000;36:279-80 Medline 